\title{
Hábitos alimentares e aconselhamento sobre alimentação saudável na atenção primária à saúde
}

\author{
Eating habits and advice on healthy eating in primary health care \\ Hábitos alimentarios y consejos sobre alimentación saludable en atención primaria de \\ salud
}

Maria Andressa Gomes Barbosa ${ }^{1 *}$, Eduarda Ângela Pessoa Cesse ${ }^{1}$, Silvia Pereira da Silva de Carvalho Melo ${ }^{1}$, Vanessa Sá Leal ${ }^{2}$, Nathália Paula de Souza², Silvana Gonçalves Brito de Arruda².

\section{RESUMO}

Objetivo: Avaliar os hábitos alimentares de usuários da atenção primária e analisar os fatores associados ao recebimento de aconselhamento sobre alimentação saudável. Métodos: Estudo transversal, analítico, realizado com 148 adultos, no município de Vitória de Santo Antão/Pernambuco, em 2016. Entre os dados coletados incluiram-se: aconselhamento sobre alimentação saudável e motivos que estimulam/dificultam à adoção de uma alimentação saudável, hábitos alimentares e aspectos comportamentais. Resultados: Houve maior adequação no consumo de feijão $(64,1 \%)$, carnes e ovos $(64,8 \%)$, alimentos fritos e embutidos $(61,4 \%)$, doces e refrigerantes $(70,9 \%)$, no hábito de retirar a gordura da carne $(95,9 \%)$, na adição de sal na hora da refeição $(92,5 \%)$ e no número de copos de água ingerido por dia $(56,7 \%)$. O consumo adequado de carnes e ovos, tipo de leite que consome, alimentos fritos e embutidos e doces/refrigerantes foi estatisticamente significante entre os que já receberam aconselhamento para uma alimentação saudável. Conclusão: A alimentação dos usuários se mostrou adequada em relação a maior parte dos itens avaliados. E os achados reforçam a importância da realização do aconselhamento sobre modos de vida saudável.

Palavras-chave: Atenção primária à saúde, Hábitos alimentares, Comportamentos saudáveis.

\begin{abstract}
Objective: Assess the eating habits of primary care users and analyze the factors associated with receiving advice on healthy eating. Methods: Cross-sectional, analytical study, carried out with 148 adults, in the municipality of Vitória de Santo Antão / Pernambuco, in 2016. Among the data collected were: advice on healthy eating and reasons that encourage / hinder the adoption of healthy eating, eating habits and behavioral aspects. Results: There was a greater adequacy in the consumption of beans (64.1\%), meat and eggs $(64.8 \%)$, fried and sausages $(61.4 \%)$, sweets and soft drinks $(70.9 \%)$, in the habit of removing the meat fat $(95.9 \%)$, the addition of salt at mealtime $(92.5 \%)$ and the number of glasses of water ingested per day $(56.7 \%)$. Adequate consumption of meat and eggs, type of milk you consume, fried and sausage foods and sweets / soft drinks was statistically significant among those who have already received advice on healthy eating. Conclusion: The users' diet proved to be adequate in relation to most of the items evaluated. And the findings reinforce the importance of carrying out counseling on healthy lifestyles.
\end{abstract}

Keywords: Primary health care, Eating habits, Healthy behaviors.

\section{RESUMEN}

Objetivo: Valorar los hábitos alimentarios de los usuarios de atención primaria y analizar los factores asociados a recibir consejos sobre alimentación saludable. Métodos: Estudio transversal, analítico, realizado con 148 adultos, en el municipio de Vitória de Santo Antão / Pernambuco, en 2016. Entre los datos recolectados se encuentran: consejos sobre alimentación saludable y razones que incentivan / dificultan la adopción de hábitos alimentarios saludables y aspectos de comportamiento. Resultados: Hubo una mayor adecuación en el consumo de frijoles (64,1\%), carnes y huevos (64,8\%), fritos y embutidos

${ }^{1}$ Fundação Oswaldo Cruz (Fiocruz), Recife - PE. *E-mail: andressa.gomes2009@hotmail.com

2 Universidade Federal de Pernambuco (UFPE), Vitória de Santo Antão - PE. 
$(61,4 \%)$, dulces y refrescos $(70,9 \%)$, en el hábito de eliminar el la grasa de la carne $(95,9 \%)$, la adición de sal en las comidas (92,5\%) y el número de vasos de agua ingeridos al día $(56,7 \%)$. El consumo adecuado de carne y huevos, tipo de leche que consume, frituras y embutidos y dulces / refrescos fue estadísticamente significativo entre los que ya recibieron consejos sobre alimentación saludable. Conclusión: La dieta de los usuarios resultó adecuada en relación con la mayoría de los ítems evaluados. Y los hallazgos refuerzan la importancia de realizar un asesoramiento sobre estilos de vida saludables.

Palabras clave: Atención primaria de salud, Hábitos alimentarios, Comportamientos saludables.

\section{INTRODUÇÃO}

A alimentação da população brasileira tem passado por transformações ao longo dos anos, havendo redução no consumo de alimentos básicos, como feijão e arroz, e aumento na ingestão de produtos industrializados. Foi identificado aumento na aquisição de biscoitos, confeitaria e refrigerantes e redução do açúcar entre a população no período entre os anos 80 e 2000 (IBGE, 2020a; LEVY RB, et al., 2012).

Em 2018, uma pesquisa de abrangência nacional mostrou que apenas um em cada quatro brasileiros consumia a quantidade de cinco porções diárias de frutas e hortaliças recomendada pela Organização Mundial da Saúde (OMS), enquanto o consumo de refrigerantes em cinco ou mais dias da semana foi observado em 14,4\% dos entrevistados (BRASIL, 2019).

Sabe-se que a alimentação contribui positivamente para a qualidade de vida mental, social e física dos indivíduos como afirma Martinez S. (2013). No entanto, a alimentação não saudável afeta negativamente a saúde da população aumentando o risco das Doenças Crônicas Não Transmissíveis (DCNT), a exemplo da hipertensão arterial sistêmica, além de ser apontada como o principal fator de risco para os Anos de Vida Ajustado por Incapacidade (DALYs - Disability Adjusted Life Years) no Brasil (MENDONÇA RD, et al., 2017; MONTEIRO CA, et al., 2018; MALTA DC, et al., 2017).

De acordo com Souza NP, et al. (2017), paralelamente ao processo de urbanização no Brasil houve um intenso avanço na produção e na oferta de alimentos industrializados, fato que contribuiu para as mudanças de hábitos alimentares na população. Estando as modificações na alimentação e no estilo de vida dos indivíduos entre os principais fatores que explicam o aumento de DCNT e morbidades, como a obesidade, que dobrou sua prevalência em mais de 70 países desde os anos 80 , característica refletida pelo fenômeno da transição nutricional e suas consequências sobre a população (AFSHIN A, et al., (2017); FERREIRA APS, et al., 2013).

Em vista desse contexto e segundo Lopes ACS, et al. (2014), o aconselhamento para realização de hábitos saudáveis de vida, como a alimentação, é considerado de grande importância para a promoção da saúde e prevenção de doenças na Atenção Primária à Saúde (APS). No estudo de Barbosa MAG, et al. (2017), verificou-se que indivíduos que participam de ações de promoção da saúde recebem mais esse tipo de aconselhamento do que os que não participam. No entanto, muitos usuários ainda buscam a assistência com foco na doença e não na prevenção de doenças e promoção da saúde (FRANCO ECD, 2012).

A importância do aconselhamento por profissionais de saúde sobre hábitos saudáveis parece favorecer práticas saudáveis entre a população. Mas Flores TR, et al. (2018), observam que é complexo o processo de adesão dos indivíduos a um estilo de vida saudável. Reforçando essa afirmativa Barbosa MAG, et al. (2017) identificaram que as condições financeiras e as restrições alimentares realizadas estão entre as dificuldades encontradas por usuários da APS para colocar em prática as informações recebidas sobre alimentação saudável. E para agravar esse cenário, ainda são constatadas orientações simplistas, proibitivas, sem diálogo e que não consideram os hábitos e contexto de vida dos indivíduos como identificado por Gomes MF, et al. (2017). Bem como, há profissionais de saúde que afirmam preferir realizar o aconselhamento sobre modos saudáveis de vida aos usuários já adoecidos, durante visitas domiciliares e de forma individual (MENDONÇA RD, et al., 2015).

Diante do exposto, é necessário aprofundar o conhecimento acerca dos hábitos alimentares e fatores relacionados ao aconselhamento sobre alimentação saudável entre a população, para que seja possível 
avaliar e aprimorar as ações de promoção da saúde de acordo com as diferentes realidades. Sendo assim, esse estudo objetivou avaliar os hábitos alimentares de usuários da atenção primária e analisar os fatores associados ao recebimento de aconselhamento sobre alimentação saudável.

\section{MÉTODOS}

Trata-se de um estudo transversal, de caráter descritivo e analítico. O qual foi executado em três Unidades de Saúde da Família (USF) e no projeto de extensão universitária "Cintura Fina" no município de Vitória de Santo Antão - PE, entre os meses de junho a outubro de 2016. O referido município esta localizado a $55 \mathrm{~km}$ da capital pernambucana e tem população estimada pelo Instituto Brasileiro de Geografia e Estatística (2019) em 138.757 habitantes. O projeto "Cintura Fina" abrangia usuários dos bairros de Água Branca e da Matriz, ofertando três vezes por semana, atividades vinculadas a atenção primária sob responsabilidade da UFPE/CAV, incluindo a prática de atividade física e promoção da alimentação saudável.

As USF incluídas nesse estudo foram selecionadas por conveniência, tendo em vista aspectos de logística. A amostra foi composta por 150 indivíduos, também selecionados por conveniência, dos quais dois se recusaram a participar da pesquisa. Foram incluídos indivíduos maiores de 20 anos que estavam presentes nas USF ou no projeto de extensão no momento da coleta de dados. Mulheres grávidas foram excluídas da amostra porque os comportamentos e hábitos alimentares nessa fase da vida diferem consideravelmente em relação às demais e as informações fornecidas poderiam se caracterizar como viés de pesquisa.

A coleta de dados foi realizada por meio da aplicação de questionário semiestruturado composto por questões socioeconômicas e demográficas (sexo, idade, escolaridade, situação de trabalho atual e renda familiar). As variáveis referentes à presença de comorbidades e o uso de medicamentos foram utilizadas para traçar o perfil clínico dos usuários. Também foram incluídas questões relacionadas aos aspectos comportamentais, aconselhamento sobre alimentação saudável e motivos que estimulam ou dificultam à adoção de uma alimentação saudável. As referidas perguntas foram baseadas em instrumento utilizados nos estudos de Toledo MTT, et al. (2013) e Andrade KA, et al. (2012), e adaptadas a realidade dos locais onde a pesquisa se realizou.

Os aspectos comportamentais foram verificados através do teste "Como está sua alimentação?" proposto pelo Ministério da Saúde na versão de bolso do primeiro Guia Alimentar para a População Brasileira. O questionário contempla 18 questões sobre hábitos alimentares, consumo de bebida alcoólica, prática regular de atividade física e leitura de rótulos alimentares, as quais foram avaliadas com base nas recomendações do referido guia em sua versão completa e classificadas como adequada ou inadequada (BRASIL, 2008).

Foi considerado consumo adequado: 3 ou mais porções de frutas/dia; 4 ou mais colheres de sopa/dia de legumes e verduras; 2 ou mais colheres/sopa/dia de feijão, lentilha, ervilha, grão-de-bico, soja, fava, sementes ou castanhas; 1 ou 2 porções de carnes ou ovos/dia; o hábito de retirar a gordura/pele de carne ou aves; 3 ou mais porções/dia de leite ou derivados; o consumo de leite com baixo teor de gorduras; menor que 2 vezes/semana, raro ou nunca de alimentos fritos e embutidos e doces e refrigerantes; a utilização de óleos vegetais no preparo dos alimentos; não colocar mais sal no alimento quando já está no prato; a ingestão de 6 ou mais copo de água/dia; o consumo eventual, raro ou não consumo de bebidas alcoólicas. A prática de atividade física diária de pelo menos 30 minutos, e a leitura algumas vezes, sempre ou quase sempre da informação nutricional dos rótulos dos alimentos também foram considerados como adequados. As demais opções de respostas para cada pergunta do questionário foram consideradas como inadequadas.

Para chegar ao resultado final do teste "Como está sua alimentação?" a cada uma das respostas era atribuída uma pontuação específica. Ao final, somava-se os pontos obtidos pelas respostas e quando o valor atingia até 28 pontos o indivíduo era classificado como 'Você precisa tornar sua alimentação e seus hábitos de vida mais saudáveis!'; entre 29 e 42 pontos como alguém que deve 'ficar atento com sua 
alimentação e outros hábitos como atividade física e consumo de líquidos' e quando o valor era superior a 43 pontos recebia o 'Parabéns! Você está no caminho para o modo de vida saudável'. Para termos de análises do presente estudo os indivíduos foram agrupados em duas categorias, os que obtiveram pontuação de até 42 pontos foram classificados como "Precisa melhorar" e os de pontuação superior em "Parabéns".

Foi considerada como variável dependente a condição de ter recebido (ou não) algum aconselhamento sobre alimentação saudável. Este foi autorreferido pelos entrevistados e recebido por algum profissional, sendo eles da estratégia de saúde da família (médico, enfermeiro, agente comunitário de saúde, dentista), nutricionista ou outros.

A percepção dos usuários quanto aos motivos que estimulam e dificultam a alimentação saudável foi identificada através das seguintes perguntas: "Qual a maior dificuldade que você encontra para seguir as orientações recebidas?" e "Qual (is) motivo (s) o estimula a praticar uma alimentação saudável?". As respostas foram classificadas em categorias, seguindo a técnica de análise de conteúdo, a qual consiste nas seguintes etapas: pré-análise, exploração do material, tratamento dos resultados e interpretação (BARDIN L, 1977).

Inicialmente, foram realizadas análises descritivas a fim de caracterizar a distribuição de frequência das variáveis em estudo. Para se determinar a associação entre as variáveis independentes e o aconselhamento sobre alimentação saudável utilizou-se o Teste Qui-Quadrado de Pearson, adotando o nível de significância $p<0,05$. A análise dos dados foi realizada com auxílio do programa SPSS 20.0 (SPSS Inc., Chicago, Estados Unidos).

O estudo foi aprovado pelo Comitê de Ética e Pesquisa do Centro de Ciências da Saúde da Universidade Federal de Pernambuco (Parecer №. 1.599.787). Todos os que participaram desta pesquisa assinaram o Termo de Consentimento Livre e Esclarecido (TCLE).

\section{RESULTADOS}

No que diz respeito às características socioeconômicas, demográficas e clínicas dos participantes, constatou-se que a maior parte da amostra foi do sexo feminino $(93,2 \%)$, apresentava idade $\geq 60$ anos $(39,2 \%)$, tinha até 8 anos de estudo $(73,6 \%)$, não trabalhava no momento da entrevista $(85,1 \%)$ e relatou uma renda familiar de até 1 salário mínimo (57,4\%), o equivalente a $R \$ 880,00$ no período da pesquisa. Quanto aos aspectos clínicos a presença de comorbidades foi relatada por $68,2 \%$ e o uso contínuo de medicamentos por $64,9 \%$ dos usuários (Tabela 1 ).

Quanto aos hábitos alimentares, foi identificada maior frequência de adequação no consumo de feijão $(64,1 \%)$, de carnes e ovos $(64,8 \%)$, de alimentos fritos e embutidos $(61,4 \%)$ e de doces e refrigerantes $(70,9 \%)$. Bem como no hábito de retirar a gordura da carne $(95,9 \%)$, na adição de sal na hora da refeição $(92,5 \%)$ e no número de copos de água ingerido por dia (56,7\%). Observou-se predominância de inadequação no consumo de frutas $(74,3 \%)$, de verduras/legumes $(64,8 \%)$, de leite e derivados $(85,8 \%)$ e na leitura da informação nutricional no rótulo dos alimentos (78,3\%) (Tabela 2).

Entre a população estudada 59\% referiu já ter recebido aconselhamento sobre alimentação saudável. Em relação aos possíveis fatores associados a receber esse aconselhamento, a idade se mostrou estatisticamente significante ao desfecho com um aumento progressivo com o avançar da idade $(p<0,001)$. Além disso, indvíuos que não trabalhavam $(p=0,042)$ e que faziam uso de medicameto $(p<0,001)$ tinham mais chance de receberem aconselhamento (Tabela 3).

Quanto aos hábitos alimentares foi estatisticamente significante o consumo adequado de carnes e ovos $(p=0,018)$, tipo de leite que consome $(p=0,002)$, alimentos fritos e embutidos $(p=0,037)$, doces e refrigerantes $(p<0,001)$ entre os que já foram aconselhados para uma alimentação saudável. $O$ aconselhamento foi maior também entre os que tinham prática regular de atividade física adequada $(p=0,034)$ e entre os que estavam na categoria "Parabéns" $(p=0,037)$ do resultado do teste "Como está sua alimentação?" (Tabela 3). 
Tabela 1 - Perfil socioeconômico, demográfico e clínico de usuários do Sistema Único de Saúde, 2016.

\begin{tabular}{lc}
\hline VARIÁVEL & $\mathbf{N}(\%)$ \\
\hline Sexo & $10(6,8)$ \\
\hline Masculino & $138(93,2)$ \\
\hline Feminino & $36(24,3)$ \\
\hline Idade & $54(36,5)$ \\
\hline 21 a 39 anos 59 anos & $58(39,2)$ \\
60 ou mais anos & $109(73,6)$ \\
\hline Escolaridade & $39(26,4)$ \\
\hline Até 8 anos de estudos & $22(14,8)$ \\
Mais de 8 anos de estudos & $126(85,1)$ \\
\hline Trabalha atualmente & $85(57,4)$ \\
\hline Sim & $48(32,4)$ \\
Não & $15(10,1)$ \\
\hline Renda familiar mensal & \\
\hline Até 1 SM & $101(68,2)$ \\
Acima de 1 e abaixo de 2 SM & $47(31,8)$ \\
Acima de 2 SM & $96(64,9)$ \\
\hline ASPECTOS CLíNICOS & $52(35,1)$ \\
\hline Apresenta comorbidade & $\mathbf{1 4 8}(100)$ \\
\hline Sim & \\
Não & \\
\hline Uso contínuo de medicamentos & \\
\hline Sim & \\
Não & \\
\hline TOTAL: & \\
\hline Legenda: N Frequencia absla & \\
\hline
\end{tabular}

Legenda: $\mathrm{N}$ - Frequência absoluta; \% - Frequência relativa; SM= Salário Mínimo. Fonte: Barbosa MAG, et al., 2020. 
Tabela 2 - Aspectos comportamentais de usuários do Sistema Único de Saúde, 2016.

\begin{tabular}{|c|c|}
\hline VARIÁVEL & $\mathbf{N}(\%)$ \\
\hline \multicolumn{2}{|l|}{ Frutas } \\
\hline Adequado & $38(25,6)$ \\
\hline Inadequado & $110(74,3)$ \\
\hline \multicolumn{2}{|c|}{ Legumes e verduras } \\
\hline Adequado & $52(35,1)$ \\
\hline Inadequado & $96(64,8)$ \\
\hline \multicolumn{2}{|c|}{ Carnes e ovos } \\
\hline Adequado & $96(64,8)$ \\
\hline Inadequado & $52(35,1)$ \\
\hline \multicolumn{2}{|c|}{ Tira a gordura da carne } \\
\hline Adequado & $142(95,9)$ \\
\hline Inadequado & $6(4,0)$ \\
\hline \multicolumn{2}{|c|}{ Leite e derivados } \\
\hline Adequado & $21(14,1)$ \\
\hline Inadequado & $127(85,8)$ \\
\hline \multicolumn{2}{|l|}{ Feijão } \\
\hline Adequado & $95(64,1)$ \\
\hline Inadequado & $53(35,8)$ \\
\hline \multicolumn{2}{|c|}{ Tipo de leite que consome ${ }^{\star}$} \\
\hline Adequado & $48(35,5)$ \\
\hline Inadequado & $95(66,4)$ \\
\hline \multicolumn{2}{|c|}{ Alimentos fritos e embutidos } \\
\hline Adequado & $91(61,4)$ \\
\hline Inadequado & $57(38,5)$ \\
\hline \multicolumn{2}{|c|}{ Doces e refrigerantes } \\
\hline Adequado & $105(70,9)$ \\
\hline Inadequado & $43(29,0)$ \\
\hline \multicolumn{2}{|c|}{ Tipo de gordura que utiliza } \\
\hline Adequado & $106(71,6)$ \\
\hline Inadequado & $42(28,3)$ \\
\hline \multicolumn{2}{|c|}{ Adição de sal na hora da refeição } \\
\hline Adequado & $137(92,5)$ \\
\hline Inadequado & $11(7,4)$ \\
\hline \multicolumn{2}{|c|}{ Copos de água ingerido por dia } \\
\hline Adequado & $84(56,7)$ \\
\hline Inadequado & $64(43,2)$ \\
\hline \multicolumn{2}{|c|}{ Consumo de bebidas alcoolicas } \\
\hline Adequado & $147(99,3)$ \\
\hline Inadequado & $1(0,6)$ \\
\hline \multicolumn{2}{|c|}{ Prática regular de atividade física } \\
\hline Adequado & $112(75,6)$ \\
\hline Inadequado & $36(24,3)$ \\
\hline \multicolumn{2}{|c|}{ Leitura da informação nutricional no rótulo dos alimentos ${ }^{\star \star}$} \\
\hline Adequado & $26(21,6)$ \\
\hline Inadequado & $94(78,3)$ \\
\hline TOTAL & $148(100)$ \\
\hline
\end{tabular}

Legenda: $N$ - Frequência absoluta; \% - Frequência relativa; $S M=$ Salário Mínimo; ${ }^{\star} n=143 ;{ }^{* *} n=120$. Fonte: Barbosa MAG, et al., 2020. 
Tabela 3 - Variáveis associadas ao aconselhamento sobre alimentação saudável entre usuários do Sistema Único de Saúde, 2016.

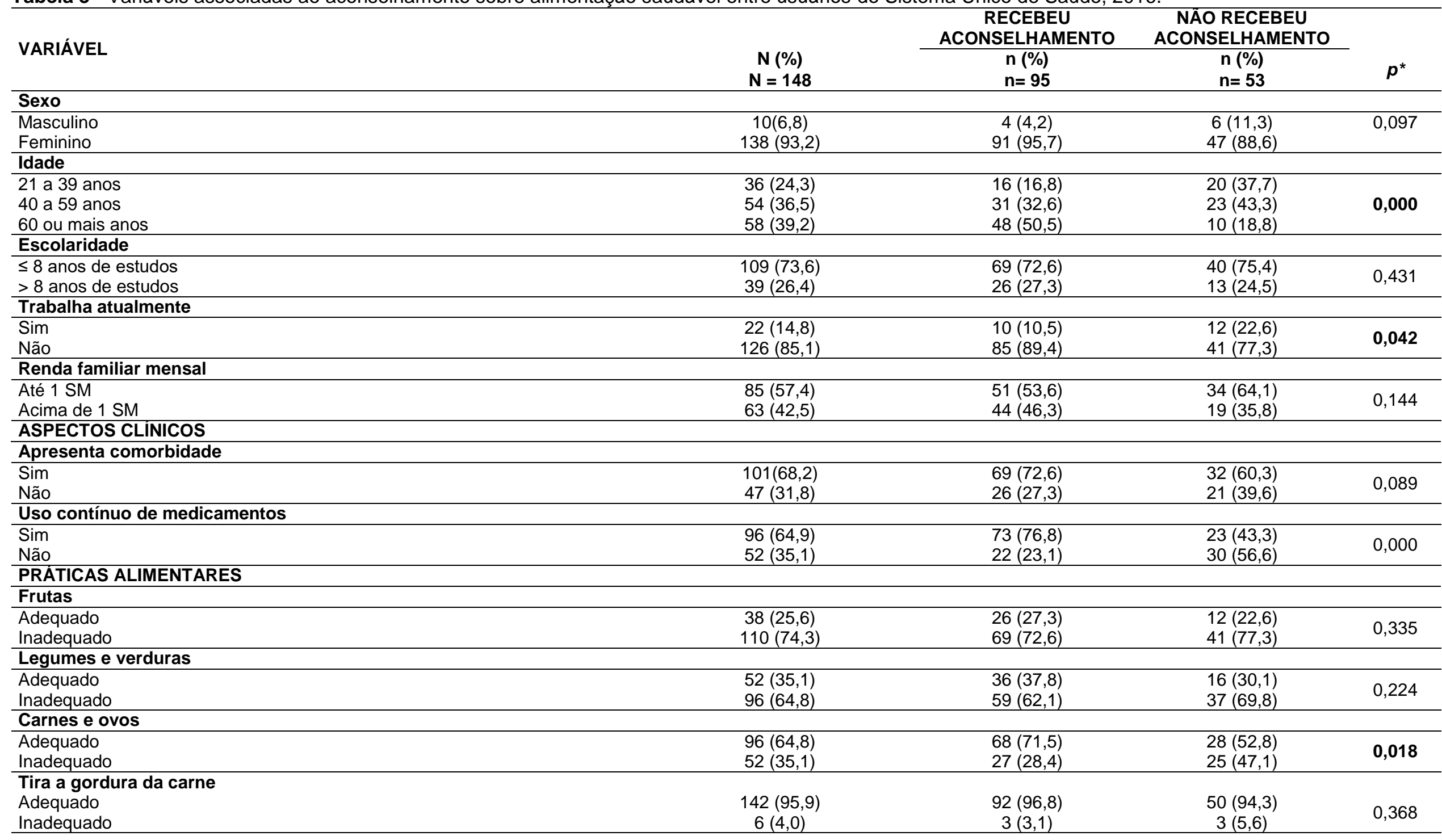




\begin{tabular}{|c|c|c|c|c|}
\hline VARIÁVEL & $\begin{array}{c}N(\%) \\
N=148\end{array}$ & $\begin{array}{c}\text { RECEBEU } \\
\text { ACONSELHAMENTO } \\
\mathrm{n}(\%) \\
\mathrm{n}=95\end{array}$ & $\begin{array}{c}\text { NÄO RECEBEU } \\
\text { ACONSELHAMENTO } \\
\mathrm{n}(\%) \\
\mathrm{n}=53\end{array}$ & $p^{*}$ \\
\hline \multicolumn{5}{|l|}{ Leite e derivados } \\
\hline $\begin{array}{l}\text { Adequado } \\
\text { Inadequado }\end{array}$ & $\begin{array}{c}21(14,1) \\
127(85,8)\end{array}$ & $\begin{array}{l}16(16,8) \\
79(83,1)\end{array}$ & $\begin{array}{c}5(9,4) \\
48(90,5)\end{array}$ & 0,161 \\
\hline \multicolumn{5}{|l|}{ Feijão } \\
\hline $\begin{array}{l}\text { Adequado } \\
\text { Inadequado }\end{array}$ & $\begin{array}{l}95(64,1) \\
53(35,8)\end{array}$ & $\begin{array}{l}58(61,0) \\
37(38,9) \\
\end{array}$ & $\begin{array}{l}37(69,8 \\
16(30,1) \\
\end{array}$ & 0,188 \\
\hline \multicolumn{5}{|c|}{ Tipo de leite que consome } \\
\hline $\begin{array}{l}\text { Adequado } \\
\text { Inadequado }\end{array}$ & $\begin{array}{l}48(35,5) \\
95(66,4)\end{array}$ & $\begin{array}{l}39(81,2) \\
53(55,8)\end{array}$ & $\begin{array}{c}9(18,8) \\
42(44,2)\end{array}$ & 0,002 \\
\hline \multicolumn{5}{|c|}{ Alimentos fritos e embutidos } \\
\hline $\begin{array}{l}\text { Adequado } \\
\text { Inadequado }\end{array}$ & $\begin{array}{l}91(61,4) \\
57(38,5) \\
\end{array}$ & $\begin{array}{l}64(67,3) \\
31(32,6) \\
\end{array}$ & $\begin{array}{l}27(50,9) \\
26(49,0) \\
\end{array}$ & 0,037 \\
\hline \multicolumn{5}{|c|}{ Doces e refrigerantes } \\
\hline $\begin{array}{l}\text { Adequado } \\
\text { Inadequado }\end{array}$ & $\begin{array}{c}105(70,9) \\
43(29,0) \\
\end{array}$ & $\begin{array}{l}79(83,1) \\
16(16,8) \\
\end{array}$ & $\begin{array}{l}26(49,0) \\
27(50,9) \\
\end{array}$ & 0,000 \\
\hline \multicolumn{5}{|c|}{ Tipo de gordura que utiliza } \\
\hline $\begin{array}{l}\text { Adequado } \\
\text { Inadequado }\end{array}$ & $\begin{array}{c}106(71,6) \\
42(28,3) \\
\end{array}$ & $\begin{array}{l}68(71,5) \\
27(28,4) \\
\end{array}$ & $\begin{array}{l}38(71,6) \\
15(28,3) \\
\end{array}$ & 0,572 \\
\hline \multicolumn{5}{|c|}{ Adição de sal na hora da refeição } \\
\hline $\begin{array}{l}\text { Adequado } \\
\text { Inadequado }\end{array}$ & $\begin{array}{c}137(92,5) \\
11(7,4) \\
\end{array}$ & $\begin{array}{c}90(94,7) \\
5(5,2) \\
\end{array}$ & $\begin{array}{c}47(88,6) \\
6(11,3) \\
\end{array}$ & 0,154 \\
\hline \multicolumn{5}{|c|}{ Copos de água ingerido por dia } \\
\hline $\begin{array}{l}\text { Adequado } \\
\text { Inadequado }\end{array}$ & $\begin{array}{l}84(56,7) \\
64(43,2)\end{array}$ & $\begin{array}{l}56(58,9) \\
39(41,0)\end{array}$ & $\begin{array}{l}28(52,8) \\
25(47,1)\end{array}$ & 0,292 \\
\hline \multicolumn{5}{|c|}{ Consumo de bebidas alcoolicas } \\
\hline $\begin{array}{l}\text { Adequado } \\
\text { Inadequado }\end{array}$ & $\begin{array}{c}147(99,3) \\
1(0,6) \\
\end{array}$ & $\begin{array}{c}94(98,9) \\
1(1,0)\end{array}$ & $\begin{array}{c}53(100,0) \\
0(0,0)\end{array}$ & 0,454 \\
\hline \multicolumn{5}{|c|}{ Prática regular de atividade física } \\
\hline $\begin{array}{l}\text { Adequado } \\
\text { Inadequado }\end{array}$ & $\begin{array}{c}112(75,6) \\
36(24,3) \\
\end{array}$ & $\begin{array}{l}77(81,0) \\
18(18,9) \\
\end{array}$ & $\begin{array}{l}35(66,0) \\
18(33,9) \\
\end{array}$ & 0,034 \\
\hline \multicolumn{5}{|c|}{ Leitura da informação nutricional no rótulo dos alimentos } \\
\hline $\begin{array}{l}\text { Adequado } \\
\text { Inadequado }\end{array}$ & $\begin{array}{l}26(21,7) \\
94(78,3)\end{array}$ & $\begin{array}{l}17(65,4) \\
57(60,6)\end{array}$ & $\begin{array}{c}9(34,6) \\
37(39,4)\end{array}$ & 0,420 \\
\hline \multicolumn{5}{|c|}{ Resultado do teste “Como está sua alimentação?" } \\
\hline $\begin{array}{l}\text { Parabéns } \\
\text { Precisa melhorar }\end{array}$ & $\begin{array}{c}36(24,3) \\
112(75,6)\end{array}$ & $\begin{array}{l}28(77,8) \\
67(59,8)\end{array}$ & $\begin{array}{c}8(22,2) \\
45(40,2)\end{array}$ & 0,037 \\
\hline
\end{tabular}

Legenda: N - Frequência absoluta; \% - Frequência relativa; *Teste Qui-Quadrado de Pearson. Fonte: Barbosa MAG, et al., 2020. 
$\mathrm{Na}$ percepção dos usuários, entre as maiores dificuldades encontradas para seguir as orientações recebidas destacou-se as restrições na alimentação (25,3\%) e condições financeiras (25,3\%). Destacaramse também respostas como falta de interesse $(7,36 \%)$ e falta de apoio dos familiares $(4,21 \%)$.

"Por que começou tirando tudo que eu gosto (lasanha, chocolate), aí nem tentei fazer" (P1).

"Não é toda hora que tem dinheiro para comprar fruta, verdura, tem que comer o que tem" (P2).

E entre os motivos que estimulavam a praticar uma alimentação saudável ressaltaram-se as seguintes respostas: para o bem da saúde $(57,9 \%)$, para ter melhor qualidade de vida/bem-estar $(12,6 \%)$, por ter problemas de saúde $(12,6 \%)$ e para perda de peso $(7,36 \%)$.

\footnotetext{
"Para ter saúde né, a idade vai chegando a gente tem que se cuidar, se amar né, tem que fazer mesmo sendo ruim" (P1).
}

"Porque a diabetes aumenta né, aí pode cortar o dedo ou a perna né" (P2).

\section{DISCUSSÃO}

Entre os principais achados verificou-se que a maioria dos entrevistados mantinha um adequado consumo de alimentos fritos e embutidos, bem como de doces e refrigerantes. Em contrapartida, um estudo realizado numa Academia da Cidade, em Belo Horizonte-MG, com 145 usuários de 20 anos ou mais de idade, identificou como característica do perfil alimentar do grupo o consumo excessivo desses tipos de alimentos (MOREIRA RAM, et al., 2012).

$\mathrm{O}$ raro consumo de embutidos e refrigerantes nesse estudo foi visto como um aspecto positivo, pois estes alimentos são considerados ultraprocessados e, portanto, conferem características desfavoráveis a qualidade nutricional da dieta. Para Louzada MLC, et al. (2015), os alimentos ultraprocessados no geral apresentam maior adição de sódio, açúcar e gorduras saturadas e menos fibras em sua composição nutricional. Portanto, o consumo desse tipo de alimento está associado ao maior risco de desenvolvimento de doenças, como a hipertensão arterial e a obesidade (MENDONÇA RD, et al., 2017; MONTEIRO CA, et al., 2018).

O hábito de retirar gordura aparente das carnes e de não adicionar sal ao alimento pronto também foi adequado entre a maioria dos entrevistados. Na pesquisa de Moreira RAM, et al., (2012) ao investigar a qualidade da dieta de acordo com o comportamento alimentar dos usuários da Academia da Cidade, a maior parte dos indivíduos relatou sempre retirar a gordura aparente das carnes.

Destaca-se a importância da adoção e manutenção de hábitos alimentares saudáveis durante a vida por todos os indivíduos, na perspectiva da manutenção da saúde e prevenção de doenças, principalmente àquelas diretamente relacionadas à alimentação como é o caso das DCNT (TOLEDO MTT, et al., 2013).

Em contrapartida foi identificado que predominou entre os participantes dessa pesquisa a inadequação no consumo diário de frutas e de verduras e legumes. Característica reforçada pela pesquisa de Oliveira MS, et al. (2015), realizada em Belo Horizonte-MG, que investigou o consumo de frutas e hortaliças entre 1.255 usuários da atenção básica, onde foi encontrado um consumo abaixo das recomendações diárias por $77,5 \%$ dos entrevistados, principalmente entre aqueles do sexo masculino. Na região centro-oeste do Brasil, pesquisadores também encontraram uma baixa prevalência para o consumo adequado de frutas, legumes e verduras (FLV) (26,3\%), sendo este consumo inversamente associado ao consumo de alimentos não saudáveis (DAMIANI TF, et al., 2017).

No presente estudo também foi encontrada baixa frequência dos que fazem leitura da informação nutricional nos rótulos dos alimentos. Característica semelhante aos achados de Barbosa MAG (2019), em um estudo realizado com 397 hipertensos e diabéticos usuários da atenção primária à saúde (APS) em quatro municípios pernambucanos. Por outro lado, Lindemann IL, et al. (2016) verificaram entre usuários da atenção básica de Pelotas-RS que 70,9\% dos entrevistados faziam a leitura dos rótulos dos produtos. 
Esse hábito é muito importante para a escolha de produtos alimentares com melhor qualidade nutricional, no entanto chama-se atenção para o fato de que no Brasil as informações nutricionais, são dispostas em formatos que dificultam a prática da leitura na grande maioria dos rótulos. No ano de 2019 foi visto que as maiores taxas de analfabetismo do país se concentram na região nordeste e as menores na região sul, o que reforça a necessidade de rever a apresentação da rotulagem dos alimentos no país a fim de facilitar a compreensão e contribuir para a escolha do consumidor (IBGE, 2020b).

A maior frequência de ter recebido aconselhamento para uma alimentação saudável esteve entre os que tinham idade mais avançada, que não trabalhavam e que faziam uso contínuo de algum medicamento. Estudos apontam uma baixa frequência de recebimento de aconselhamento para modos saudáveis de vida, como alimentação e atividade física entre usuários da atenção primária a saúde, o qual ocorre principalmente entre aqueles que apresentam alguma morbidade. E alertam para a necessidade dessa prática ser realizada em caráter preventivo e como promotora da saúde vista a sua importância (LOPES ACS, et al., 2014; TOLEDO MTT, et al., 2017)

Segundo Corso LR, et al. (2017), conhecer as características dos usuários é importante uma vez que ajuda as equipes de saúde no direcionamento das orientações. Santos RP, et al. (2012) relatam que os profissionais da saúde devem conhecer o contexto no qual os usuários estão inseridos, identificar dificuldades e adequar as condutas conforme as necessidades e particularidades evitando padronizações. É preciso compreender que as representações contidas nas histórias de vida dos usuários nem sempre correspondem as normas da objetividade científica (MASSIMO EAL, et al., 2015).

$\mathrm{Na}$ presente pesquisa, os usuários que referiram ter recebido aconselhamento nutricional apresentaram maior adequação no consumo de carnes e ovo ( 1 ou 2 vezes ao dia), maior prevalência na ingestão de leite com baixo teor de gorduras, e menor consumo de frituras e embutidos, doces e refrigerantes (raro/nunca). Corroborando com Andrade KA, et al. (2012) em uma pesquisa no serviço de atenção primária em Minas Gerais, a qual evidencia que usuários aconselhados tiveram mais adequação para o consumo de alimentos como ovos, refrigerantes, salgados e balas/goma de mascar. Para Toledo MTT, et al. (2017), o aconselhamento é um meio de promoção da saúde e de construção de modos saudáveis de vida. Sendo sua prática essencial, uma vez que influencia favoravelmente sobre o padrão alimentar e, por conseguinte, sobre a qualidade de vida e a saúde dos indivíduos (ANDRADE KA, et al., 2012).

A maior prática de atividade física também foi relatada por indivíduos que afirmaram terem sidos aconselhados. O que se aproxima aos achados de Flores TR, et al. (2018), no estudo de base populacional com idosos em Pelotas, Rio Grande do Sul, ratificando a importância dessas orientações para a promoção de um estilo de vida mais saudável. Pesquisadores encontraram baixa prevalência de aconselhamento para prática de atividade física em UBSs do mesmo município, o qual esteve associado ao aumento da idade, à presença de comorbidades, ao uso contínuo de medicamentos e à quantidade de vezes que o indivíduo esteve na UBS no último ano. Os autores ressaltam que mesmo com baixa frequência essa prática influenciou positivamente nos estágios de mudança de comportamento para prática de atividade física entre os indivíduos (HAFELE V e SIQUEIRA FV, 2016).

Por fim, no topo das dificuldades relatadas pelos participantes do nosso estudo para seguir as orientações recebidas estiveram as restrições alimentares e as condições financeiras. Sabe-se que diversos são os motivos que dificultam essa adesão. Entre as barreiras encontradas na literatura estão a falta de tempo $(27,5 \%)$ e a necessidade de mudança de hábitos (23\%), bem como o baixo poder aquisitivo, o preço, a preguiça e a carência de iniciativas públicas, o conhecimento insuficiente, a necessidade de abrir mão de alimentos considerados não saudáveis, o custo elevado dos alimentos considerados saudáveis $(57,6 \%)$ e a força de vontade insuficiente (SANTOS RP, et al., 2012; LINDEMANN IL, et al., 2016).

Todavia, entre os motivos que facilitavam a adoção de uma alimentação saudável, a questão da saúde foi a mais citada por nossos entrevistados. Fator mais citado também como promotor do consumo de frutas e hortaliças em um estudo realizado em polos da academia da saúde em Belo Horizonte, Minas Gerais, o qual identificou também outros fatores como a melhoria da situação financeira, gostar, criação e origem familiar, prevenção/controle de doenças, hortaliça ser considerada como parte da refeição, comércio próximo e estratégias de compra (FIGUEIRA TR, et al., 2016). 
Entre as limitações para as análises estatísticas do presente estudo destaca-se o tamanho da amostra e o delineamento transversal. No entanto, ressalta-se a relevância de identificar aspectos alimentares e comportamentais de frequentadores de serviços de saúde o que possibilita a reflexão e o aperfeiçoamento de estratégias de promoção da saúde.

\section{CONCLUSÃO}

A alimentação dos usuários da atenção primária se mostrou adequada em relação a maior parte dos itens avaliados. Mas ainda assim, o consumo de frutas, verduras/legumes e de leites e derivados foram considerados inadequados. A idade mais avançada, não trabalhar e fazer uso contínuo de algum medicamento foram diretamente associados a ter recebido aconselhamento sobre alimentação saudável. $\mathrm{E}$ entre aqueles que receberam esse aconselhamento foi significativamente mais adequado o consumo de carnes e ovos, de alimentos fritos e embutidos, de doces e refrigerantes, do tipo de leite consumido, bem como a prática regular de atividade física. Os achados reforçam a importância da realização do aconselhamento sobre modos de vida saudável para adoção destes por parte da população. Bem como propõe a reflexão para a necessidade do desenvolvimento de estratégias de promoção da saúde entre os indivíduos, com atenção especial aos jovens, ativos no mercado de trabalho e que não fazem uso de medicamentos, os quais parecem não procurar com frequência os serviços de saúde.

\section{REFERÊNCIAS}

1. AFSHIN, A. et al. Health Effects of Overweight and Obesity in 195 Countries over 25 Years, The New Englad Journal of Medicine, 2017; 377(1): 13-27.

2. ANDRADE KA, et al. Aconselhamento sobre modos saudáveis de vida na Atenção Primária e práticas alimentares dos usuários. Revista da Escola de Enfermagem da USP, 2012; 46(5): 1117-1124.

3. BARBOSA MAG, et al. Participação de usuários da atenção primária em práticas de promoção da saúde. Revista Brasileira em Promoção da Saúde, 2017; 30(4): 1-11.

4. BARBOSA MAG. Consumo alimentar de hipertensos e diabéticos: um olhar sob a perspectiva do processamento industrial dos alimentos. Dissertação (Mestrado Acadêmico em Saúde Pública) - Instituto Aggeu Magalhães. Fundação Oswaldo Cruz, Recife, 2019; 102 p.

5. BARDIN L. Análise de conteúdo. Lisboa: Edições 70; 1977.

6. BRASIL. Ministério da Saúde. Guia alimentar para a população brasileira: promovendo a alimentação saudável. Brasília: Ministério da Saúde; 2008.

7. BRASIL. Ministério da Saúde (MS). Vigitel Brasil 2018: vigilância de fatores de risco e proteção para doenças crônicas por inquérito telefônico. Brasília: MS. 2019.

8. CORSO LR, et al. Alimentação equilibrada: uma análise dos hábitos alimentres de portadores de diabetes melittus. Revista Uningá. 52 (1): 44-50.

9. DAMIANI TF, et al. Consumo de frutas, legumes e verduras na Região Centro-Oeste do Brasil: prevalência e fatores associados. Ciência \& Saúde Coletiva, 2017; 22(2):369-382.

10. FERREIRA APS, et al. Prevalência e fatores associados da obesidade na população brasileira: estudo com dados aferidos da Pesquisa Nacional de Saúde, Revista Brasileira de Epidemiologia, 2013; 22: 1980-5497.

11. FIGUEIRA TR, et al. Barreiras e fatores promotores do consumo de frutas e hortaliças entre usuários do Programa Academia da Saúde. Revista de Nutrição, 2016; 29(1): 85-95.

12. FLORES TR, et al. Aconselhamento por profissionais de saúde e comportamentos saudáveis entre idosos: estudo de base populacional em Pelotas, sul do Brasil, 2014. Epidemiologia e Serviços de Saúde,2018; 27(1): e201720112.

13. FRANCO ECD. A estratégia de saúde da família na perspectiva do usuário. Revista de Enfermagem UFSM, 2012;2(1): 49-58.

14. GOMES MF, et al. Orientações sobre alimentação ofertadas por profissionais da estratégia de saúde da família durante as consultas aos hipertensos e diabéticos. Revista de Atenção Primária à Saúde, 2017; 20(2): 203-211.

15. HAFELE V, SIQUEIRA VF. Aconselhamento para atividade física e mudança de comportamento em Unidades Básicas de Saúde. Revista Brasileira de Atividade Física e Saúde, 2016; 21(6): 581-592.

16. INSTITUTO BRASILEIRO DE GEOGRAFIA E ESTATÍSTICA (IBGE). Estimativas populacionais para os municípios e para as Unidades da Federação brasileiros em 01.07.2019.

17. INSTITUTO BRASILEIRO DE GEOGRAFIA E ESTATÍSTICA (IBGE). Pesquisa de orçamentos familiares 2017-2018: avaliação nutricional da disponibilidade domiciliar de alimentos no Brasil. Rio de Janeiro: IBGE; 2020 a.

18. INSTITUTO BRASILEIRO DE GEOGRAFIA E ESTATÍSTICA (IBGE), Diretoria de Pesquisas, Coordenação de Trabalho e Rendimento, Pesquisa Nacional por Amostra de Domicílios Contínua 2019. 2020 b.

19. LEVY RB, et al. Distribuição regional e socioeconômica da disponibilidade domiciliar de alimentos no Brasil em 2008 2009. Revista de Saúde Pública, 2012; 46(1): 6-15.

20. LINDEMANN IL, et al. Dificuldades para alimentação saudável entre usuários da atenção básica em saúde e fatores associados. Ciência \& Saúde Coletiva, 2016. 21(2): 599-610. 
21. LOPES ACS, et al. Condições de saúde e aconselhamento sobre alimentação e atividade física na Atenção Primária à Saúde de Belo Horizonte-MG. Epidemiologia e Serviços de Saúde, 2014;23(3): 475-486.

22. LOUZADA MLC, et al. Alimentos ultraprocessados e perfil nutricional da dieta no Brasil. Revista de Saúde Pública, 2015; 49(38): 1-11.

23. MALTA DC, et al. Fatores de risco relacionados à carga global de doença do Brasil e Unidades Federadas. Revista Brasileira de Epidemiologia, 2017; 20(sup.1): 217-232.

24. MARTINEZ S. A nutrição e a alimentação como pilares dos programas de promoção da saúde e qualidade de vida nas organizações. O Mundo da Saúde, 2013; 37(2): 201-207.

25. MASSIMO EAL, et al. Doenças crônicas não transmissíveis, risco e promoção da saúde: construções sociais de participantes do Vigitel. Ciência \& Saúde Coletiva, 2015; 20(3): 679-688.

26. MENDONÇA RD, et al. Incentivo à prática de aconselhamento sobre modos saudáveis de vida na Atenção Primária à Saúde Escola Anna Nery Revista de Enfermagem, 2015; 19(1): 140-146.

27. MENDONÇA RD, et al. Ultraprocessed food consumption and the incidence of hypertension in a Mediterranean cohort: the Seguimiento Universidad de Navarra Project. American Journal of Hypertension, 2017; 30(4): $358-366$.

28. MONTEIRO CA, et al. Household availability of ultra-processed foods and obesity in nineteen European countries. Public Health Nutrition, 2018; 21(1): 18-26.

29. MOREIRA RAM, et al. A qualidade da dieta de usuários de serviço de promoção da saúde difere segundo o comportamento alimentar obtido pelo modelo transteóricoRevista de Nutrição, 2012; 25(6): 719-730.

30. OLIVEIRA MS, et al. Consumo de frutas e hortaliças e as condições de saúde de homens e mulheres atendidos na atenção primária à saúde. Ciência \& Saúde Coletiva, 2015; 20(8): 2313-2322.

31. SANTOS RP, et al. Aconselhamento sobre alimentação e atividade física: prática e adesão de usuários da atenção primária. Rev Gaúch Enferm, 2012; 33(4): 14-21.

32. SOUZA NP, et al. A (des)nutrição e o novo padrão epidemiológico em um contexto de desenvolvimento e desigualdades, Ciência \& Saúde Coletiva, 2017; 22(7): 2257-2266.

33. TOLEDO MTT, et al. Aconselhamento sobre modos saudáveis de vida na Atenção Primária à Saúde. O Mundo da Saúde, 2017; 41(1): 87-97.

34. TOLEDO MTT, et al. Adesão a modos saudáveis de vida mediante aconselhamento por profissionais de saúde. Rev Saúde Pública, 2013; 47(3): 540-548. 